\title{
Archivos de inteligencia: imaginarios, saberes, sentidos. La experiencia de la CPM a 20 años de la cesión del archivo de la DIPPBA
}

\author{
Intelligence archives: imaginary, knowledge, meanings. The experience of the \\ CPM after 20 years of the transfer of the DIPPBA archive
}

\author{
Julieta Sahade \\ julietasahade@gmail.com \\ Comisión Provincial por la Memoria (CPM), \\ Argentina
}

Ingrid Jaschek

ingridjaschek@gmail.com

Comisión Provincial por la Memoria (CPM),

Argentina

Magdalena Lanteri
magdalenalanteri@gmail.com
Comisión Provincial por la Memoria (CPM) /
Universidad Nacional de La Plata (UNLP), Argentina

Recepción: 19 Abril 2021

Aprobación: 17 Mayo 2021

Publicación: 01 Junio 2021

Cita sugerida: Sahade, J., Jaschek, I. y Lanteri, M. (2021). Archivos de inteligencia: imaginarios, saberes, sentidos. La experiencia de la CPM a 20 años de la cesión del archivo de la DIPPBA. Aletheia, 11(22), e086. https://doi.org/10.24215/18533701e086
Resumen: Cuando en el año 2000 el fondo documental de la Dirección de Inteligencia de la Policía de la Provincia de Buenos Aires (DIPPBA) es cedido a la Comisión por la Memoria se generó una enorme expectativa en relación con la posibilidad de encontrar allí evidencias del accionar clandestino ejercido por el Estado durante el período 1976-1983. Dichas expectativas se enmarcaron en un contexto de ausencia de políticas transparentes en materia de archivos, en las leyes de impunidad vigentes y en el proceso inédito que implicó la desclasificación de un fondo de origen reservado. Hace 17 años que el archivo de la DIPPBA está abierto a la consulta pública. El abordaje desde la disciplina archivística permitió un conocimiento integral del fondo y de la entidad productora y el reconocimiento de la inteligencia como un importante dispositivo del terrorismo de Estado. Este trabajo presentará algunas afirmaciones e interrogantes que pretenden avanzar en el conocimiento de los contextos de producción de los documentos, tensionando representaciones en torno a lo que se entiende por archivo de inteligencia.

Palabras clave: Inteligencia, Represión, Archivística, DIPPBA, CPM.

\begin{abstract}
When in 2000 the fond of the Intelligence Directorate of the Police of the Province of Buenos Aires (DIPPBA) was transferred to the Comisión por la Memoria, a huge expectation was generated in relation to the possibility of finding there evidence of clandestine actions exercised by the State during the period 1976-1983. Such expectations were framed in a context of the absence of transparent policies regarding archives, in the impunity of the prevailing laws and in the unprecedented process that implied the declassification of a confidential fund. The DIPPBA archive has been open for public consultation for 17 years. The approach from the archival discipline allowed a comprehensive knowledge of the fund, the record creator and the recognition of intelligence as an important device of State terrorism. This work will present some statements and questions to move forward in the knowledge of the contexts of document production, straining representations around what is understood by an intelligence file.
\end{abstract}

Keywords: Intelligence, Repression, Archival science, DIPPBA, CPM. 


\section{INTRODUCCIÓN}

La Comisión por la Memoria de la Provincia de Buenos Aires gestiona desde el año 2000 el fondo de la Dirección de Inteligencia de la Policía de la Provincia de Buenos Aires, el primer archivo de inteligencia de origen reservado desclasificado y abierto a la consulta pública en nuestro país.

La DIPPBA ${ }^{1}$ se crea en 1956 y el 30 de abril de 1998 -en el marco de una reforma de la policía y una intervención civil a una institución en creciente desprestigio y vinculación a hechos delictivos- es disuelta por resolución No 9 del Ministerio de Seguridad y Justicia de la Provincia de Buenos Aires. Así finalizaba el ciclo de una Dirección de Inteligencia que, con variantes a través de los años, mantuvo una estructura similar y se constituyó en una institución que realizó inteligencia político-ideológica durante toda la segunda mitad del siglo XX.

En 1999, la Cámara Federal de Apelaciones de La Plata dictó una medida cautelar de no innovar sobre el archivo de la DIPPBA a fin de asegurar su preservación por considerar que los documentos allí existentes podrían aportar pruebas para los Juicios por la Verdad que habían comenzado unos meses antes.

En diciembre del año 2000, a través de la Ley Provincial 12642/2000, la legislatura desclasificó el archivo y lo transfirió -junto al edificio donde funcionaba la DIPPBA- a la Comisión Provincial por la Memoria para que hiciera de éste un "centro de información con acceso público tanto para los afectados directos como para todo interesado en desarrollar tareas de investigación y difusión”. ${ }^{2}$

En el año 2003, la Cámara Federal de Apelaciones levantó parcialmente la medida de No Innovar, manteniéndola hasta el día de hoy solo sobre la documentación producida entre 1976-1983. En octubre de ese año, el fondo documental se abrió a la consulta pública convirtiéndose en una experiencia pionera en Argentina (Nazar, 2018). La apertura del archivo fue precedida por un intenso debate al interior de la CPM en torno a las condiciones de acceso: democratizar y reparar sin vulnerar a las víctimas. La búsqueda del equilibrio entre dar a conocer a la sociedad en su conjunto esos documentos y el resguardo de la intimidad de las personas víctimas de la persecución policial, fue lo que guió desde un principio la tarea de apertura del archivo (Jaschek, Lanteri, Sahade y Soler, 2018). Fue la primera experiencia en nuestro país de un archivo de inteligencia con documentos de origen reservado, secreto y confidencial, desclasificado y abierto a la consulta pública. $^{3}$

\section{Apertura, Sesgos, imaginarios}

$\mathrm{Al}$ acto de apertura pública del archivo asistieron más de cien personas. Fue reseñado en diarios de tirada local y nacional (Ginzberg, 2003), contó con la presencia de Adolfo Pérez Esquivel (presidente de la CPM) y Carlos Lafforgue (por entonces, Secretario Ejecutivo del Archivo Nacional de la Memoria) entre otres funcionaries y personalidades. En forma simbólica y política se le hizo entrega de copias de documentación que la DIPPBA había elaborado y archivado sobre ellas a distintas personalidades del ámbito político, cultural y social. Así fue que Madres de Plaza de Mayo Línea Fundadora y de La Plata, Abuelas, Familiares, SERPAJ, CELS, APDH La Plata, la Liga Argentina por los DDHH, la Asociación Miguel Bru, CTA, ATE, la Asociación Judicial Bonaerense, Ferroviarios, Tito Cossa fueron distinguides. En esa selección se buscó una diversidad que pudiese dar cuenta de la amplitud temporal y de perspectivas que la Dirección de Inteligencia tenía entre sus misiones y funciones, así como pensar la reparación no solo desde lo individual sino fundamentalmente desde lo colectivo: las entregas fueron mayoritariamente a organizaciones 
sociales, gremiales, políticas, de derechos humanos, y los documentos daban cuenta de actividades y acciones colectivas. Pese a ello, ya quedaba sellado el vínculo que unía casi exclusivamente el funcionamiento de la DIPPBA con el período 1976-1983 y con las violaciones a los DDHH ocurridas en la década de los 70. ${ }^{4}$

En ese entonces seguían vigentes las leyes de impunidad que impedían los procesos penales a los responsables de los crímenes de lesa humanidad, y las expectativas de familiares y organismos de DDHH en relación con la posibilidad de estar frente a un archivo que pudiese dar respuestas concretas por los crímenes perpetrados por el Estado durante la última dictadura fueron tan fuertes, que explican en parte las condiciones de posibilidad del proceso inédito de desclasificación y apertura al público de un archivo de inteligencia. ${ }^{5}$ Pero al mismo tiempo, construyeron un sesgo de sentidos e imaginarios distorsionados que tendieron a identificar fuertemente el fondo DIPPBA como un archivo de la represión clandestina durante la última dictadura militar -que excedía la categorización de los archivos de inteligencia como archivos de la represión dado por el Grupo de Expertos del ICA-UNESCO (González Quintana, 1998)- esperando que pudiese responder a determinadas búsquedas, preguntas, verdades.

¿Decían esos documentos qué había pasado con sus familiares? ¿Marcaban o señalaban a les responsables de los crímenes? ¿Estaba explícito el circuito clandestino de la represión? ¿qué información sobre las víctimas del terrorismo de Estado podía encontrarse en los documentos que conforman el fondo? ¿fue posible asumir que en esos documentos no se encontraba la verdad sino un relato, una mirada oficial/estatal/policial?

Hoy, al historizar el proceso de configuración de sentidos alrededor del fondo DIPPBA, resulta posible entender que se trató de una construcción polifónica entre la CPM, organismos de DDHH, familiares, instituciones, colectivos y personas cercanas al proceso de cesión, gestión y apertura. Un proceso en el que hubo tensiones a las que no fue ajena la propia CPM dada la diversidad de sus integrantes. La decisión política institucional fue abrir el archivo prioritariamente a las víctimas. ¿A quiénes incluía y excluía esa figura de la víctima? ¿Era posible, era el momento, era legítimo pensar en otras víctimas que no fuesen desaparecides, muertes, familiares, ex detenides?

La estructura de sentidos que se fue erigiendo en torno al fondo DIPPBA entonces, no tuvo que ver sólo con el "archivo" sino con la propia historia de la CPM, con el recorrido de los organismos, la justicia, la impunidad y las posibilidades de justicia que empezaban a vislumbrarse nuevamente.

Aquellos imaginarios y sesgos requerían difundir las características del fondo para no generar falsas expectativas, complejizando aquello que inicialmente fue comprendido como archivo de la represión.

\section{DESANDAR SENTIDOS, CONSTRUIR SABERES}

El abordaje del archivo desde la disciplina archivística -campo de saberes y herramientas- permitió un conocimiento integral del fondo y de la institución productora que, junto a la interacción con usuaries de diferentes ámbitos y al intercambio con instituciones y equipos de trabajo que gestionan fondos de fuerzas de seguridad, habilitaron nuevas/diferentes miradas, perspectivas e interrogantes que abrieron el juego a problematizar esos sentidos primigenios.

De esa manera, la idea de "archivo de la represión" lejos de desaparecer se complejiza y asume nuevos sentidos (González Quintana, 1998; Da Silva Catela y Jelin, 2002, Kahan, 2007). La primera complejidad parte de concebir a la DIPPBA en tanto entramado político-burocrático provincial pero al mismo tiempo formando parte de la comunidad informativa y en articulación con las otras Direcciones de Investigación y Seguridad de la policía provincial. ${ }^{6}$ Por otro lado, la mirada sobre las propias instituciones de inteligencia en tanto piezas constitutivas del Estado moderno -en coyunturas dictatoriales y en democracia- y sobre el papel que la inteligencia cumplió en el entramado represivo estatal durante el terrorismo de Estado.

Fue posible entonces repensar el archivo en el marco de la institución que lo produjo y leer múltiples inscripciones e imbricaciones en diferentes tramas: la administración de los poderes estatales (provincial y 
nacional), la propia estructura de las fuerzas de seguridad coyunturalmente intervenidas por las FFAA, las lógicas y dinámicas propias de los organismos de inteligencia, márgenes de autonomía y/o permeabilidad a doctrinas y políticas supranacionales, entre otras.

El trabajo con el fondo desde la perspectiva archivística se inició en el año 2006 y tuvo como primer gran desafío la elaboración del cuadro de clasificación. Dicho proceso comenzó con la identificación de la División Central Archivo y Fichero (DCAyF) como una de las secciones del fondo DIPPBA que por su magnitud consideramos un subfondo. ${ }^{7}$ El archivo de la DIPPBA en su totalidad cuenta también con documentación vinculada a la Dirección Central de Inteligencia -la cual elaboraba informes que luego de cumplido su ciclo de inteligencia podían ser incorporados a la DCAyF- a personal, a logística, a la exploración de medios escritos y sala de escucha, entre otros. Identificar con claridad las áreas productoras de la documentación fue un paso fundamental para la identificación y conceptualización del fondo. ${ }^{8}$

En una primera instancia se buscó normativa referida a la DIPPBA y a la sección "Archivo" dentro del material doctrinario del fondo que había sido relevado y analizado en el 2003. Se identificaron misiones, funciones e información que facilitó el reconocimiento de secciones, subsecciones, series y subseries. Simultáneamente se revisaron libros de registro e índice y lomos de los contenedores para cotejar, elaborar nuevas preguntas y volver a revisar buscando el orden y lógica original de la documentación (Comisión Provincial por la Memoria, 2015; Sahade, Lanteri y Jaschek, 2016).

A 20 años de la cesión del fondo y a través de los distintos recorridos de una política integral en materia de gestión documental, presentaremos algunas certezas, hipótesis e interrogantes. Algunos refieren a la institución productora, otros a la documentación por ella producida y otros que buscan indagar sobre el rol de la inteligencia durante la segunda mitad del siglo XX.

Cabe aclarar inicialmente que el fondo DIPPBA heredó documentación de organismos que la precedieron en sus tareas dentro de la órbita de la Policía de la Provincia de Buenos Aires, como las antiguas secciones de "Orden Social” y "Orden Político" y la "División de Orden Público". Por esa razón, cuando referimos al fondo debemos dar cuenta de esta documentación cuya fecha extrema más antigua es 1937. Esta herencia documental habilita una serie de interrogantes que permiten historizar la inteligencia estatal en su articulado con la dinámica política, tensionando cualquier intento de vinculación simplista. En el marco de las políticas dispuestas por la autodenominada "Revolución libertadora", Desiderio Fernández Suárez -interventor de la Jefatura de la Policía de la Provincia de Buenos Aires- en 1956 manifestaba enfáticamente las diferencias existentes entre la disuelta División de Orden Público y el nuevo organismo de inteligencia policial. Hacía hincapié en el contraste absoluto en los objetivos de inteligencia entre un gobierno y otro. ${ }^{10}$ Sin embargo, el legado documental entre un organismo y otro traspasó las fronteras políticas e ideológicas. La mesa C (comunismo) del fondo DIPPBA se nutrió de numerosos legajos personales de supuestes militantes comunistas confeccionados antes de septiembre de $1955 .{ }^{11}$ En ese contexto podemos preguntarnos ¿qué se eliminó y qué se conservó? ¿qué criterios mediaron esa transferencia? ¿cómo es posible pensar las continuidades y rupturas en la lógica de la inteligencia político ideológicas entre el gobierno Peronista y aquellos emanados del golpe del 55 ?

Más allá de esa documentación heredada, la Dirección de Inteligencia, con distintas estructuras y denominaciones a lo largo de su historia, dependió orgánicamente de Jefatura de Policía al igual que otras direcciones con el mismo rango y jerarquía tales como la de Seguridad, Investigaciones, Legal y Técnica. Su función consistió en la planificación y realización de las tareas de búsqueda, reunión, calificación, difusión y registro de la información sobre ciudadanes, organizaciones y acontecimientos en el territorio bonaerense. Sin embargo, a lo largo de su historia su radio de acción sobrepasó los límites de la provincia. El archivo de la DIPPBA es revelador del alcance territorial de las tareas de inteligencia bajo su órbita que podía extenderse hacia la Capital Federal y otras zonas del país, estableciendo redes, articulando con otros organismos afines con los que compartía la información. 
Desde su creación, la DIPPBA integró la comunidad informativa, una instancia de articulación entre los distintos servicios de inteligencia de las fuerzas de seguridad y armadas para el intercambio de información (Funes y Jaschek, 2005). En el ámbito nacional, esta comunidad informativa estuvo integrada por la Secretaría de Inteligencia del Estado (SIDE), Jefatura II de Inteligencia y Batallón de Inteligencia 601 del Ejército, el Servicio de inteligencia Naval (SIN), la Dirección de inteligencia de Gendarmería Nacional (DIG), el Servicio de Inteligencia de Prefectura Naval Argentina (SIPNA), la Dirección de Inteligencia de la Policía de la Provincia de Buenos Aires (DIPBA), el Servicio de Inteligencia Aeronáutica (SIA) y la Superintendencia de Seguridad Federal.

El legajo de Referencia 12166 del 25 de enero de 1963 describe en detalle las características de la reunión de la "comunidad informativa" y permite dimensionar el articulado entre la inteligencia provincial y otros organismos afines del país. Las reuniones se realizaban todos los miércoles de cada quincena a las 10 horas; eran de carácter obligatorio y si algún miembro requería una reunión especial o de urgencia la debía requerir al presidente de la reunión. Citamos textualmente:

En cada reunión, se repasan las informaciones ya elevadas a la S.I.D.E., respondiendo a las investigaciones que se estimaron necesarias realizar en la anterior asamblea. Luego se habla por turno sobre las novedades o situaciones que se relacionen con la tarea informativa de seguridad. Se toma nota de lo que interese y se ordenan los trabajos a realizar, cuyo resultado debe ser elevado directamente a la S.I.D.E., solicitando su distribución a la Comunidad Informativa, si ya no se hizo al término de tales trabajos. ${ }^{12}$

Los servicios de inteligencia que integraban la comunidad informativa jugaron un rol clave en la identificación de les actores sociales que pudieran ser blanco del accionar represivo.

En relación con la estructura de la DIPPBA y su despliegue territorial, señalamos algunos aspectos claves para entender su funcionamiento. La dirección central se encontraba en la ciudad de La Plata desde dónde articulaba de forma orgánica y funcional con las delegaciones, muchas de las cuales funcionaban en las cabeceras de las Unidades Regionales. En 1959 las delegaciones eran Morón, Lanús, San Martín, San Nicolás, Junín, Mar del Plata, Azul y Bahía Blanca. Con posterioridad se crean las delegaciones Quilmes, Castelar, Pehuajó, Mercedes, Chascomús, Tigre, La Matanza, La Plata y Capital Federal. Periódicamente y de manera rutinaria cada Delegación de Inteligencia reunía información sobre personas, partidos políticos, sindicatos y acontecimientos de la localidad o región bajo su órbita, y la remitía a la Dirección Central donde era analizada, evaluada e incorporada al Archivo.

Durante la última dictadura militar se producen una serie de reformulaciones administrativas tendientes a incrementar la eficacia, esto es, la disponibilidad más rápida y efectiva de la información ${ }^{13}$, y una ampliación de los organismos territoriales de reunión de información, entre los que destacamos la creación de la delegación de Capital Federal en el año 1976. Ésta tendrá a su cargo el enlace con otros organismos de inteligencia y de seguridad a nivel nacional.

En relación con el circuito de la información, de manera muy genérica podemos señalar que se trataba de un proceso que podía iniciarse a partir de un acontecimiento, una sospecha, un procedimiento o un requerimiento de otro organismo. A partir de ese momento, la Dirección, más precisamente el Jefe de la Central de Inteligencia, iniciaba una "orden de búsqueda" u "orden reunión de información”. La "orden de búsqueda” recaía en la rama Búsqueda -de la central o de las delegaciones- que se encargaba de elaborar un "plan de búsqueda" consistente en producir un conocimiento que no se poseía. Para lograrlo debía reunir diferentes datos o informaciones relacionadas con el caso a trabajar. También se consultaba al Archivo y se establecían prioridades: elementos esenciales de inteligencia (EEI) y otros requerimientos de inteligencia (OIE). Los legajos agrupados en la Mesa DS (delincuente subversivo) responden en mayor medida a esta dinámica del ciclo de inteligencia. ${ }^{14}$ El legajo 7454 , por ejemplo, se inicia con un informe de inteligencia en el que se lee: 
En razón de haberse tomado conocimiento mediante el Organismo afín BATALLÓN DE INTELIGENCIA 601, que en el establecimiento educacional secundario mencionado en el epígrafe, un grupo de profesores integrarían una célula izquierdista, desarrollando actividades de adoctrinamiento y captación dentro del alumnado del mismo, este organismo realizó una prolija investigación tendiente a establecer la responsabilidad de los citados educadores arribando posteriormente a las siguientes conclusiones... ${ }^{15}$

Otra vía de inicio del ciclo de inteligencia era la vigilancia periódica y rutinaria efectuada desde las delegaciones que operaban en el territorio. Los legajos reunidos en las mesas A, B y De reflejan esta forma de vigilancia y registro constante sobre establecimientos, agrupaciones, entidades, organizaciones. Por ejemplo, la Delegación Bahía Blanca de la DIPPBA produjo cuantiosa información sobre la actividad políticoacadémica en la Universidad Nacional del Sur. Solo el legajo 1 -de la mesa A, factor Estudiantil, Bahía Blancaque corresponde al registro minucioso de las actividades político-académicas de las autoridades y órganos rectores de esa universidad, reúne 4670 fojas distribuidas en 19 tomos y cuyas fechas extremas son 1957-1993.

Independientemente del estímulo que activara el mecanismo de inteligencia, éste habilitaba el despliegue de distintos medios de obtención de información: orgánicos -delegaciones, comisarías, subcomisarias- y no orgánicos -colaboradores o confidentes, informantes y contactos. De este modo, la información se obtenía a través de distintos procedimientos: escuchas, averiguaciones, vigilancias, seguimientos, entrevistas, visitas domiciliarias clandestinas, interrogatorios, infiltración, espionaje, explotación de prensa, detenciones.

La información obtenida era procesada, valorada -según el grado de confiabilidad de la fuente y el grado de consistencia de la información- interpretada y difundida a la comunidad informativa u otros órganos de seguridad. Parte de esos procedimientos administrativos eran archivados. Regularmente esa documentación archivada, era consultada a partir de nuevos requerimientos internos o de la comunidad informativa.

La División Central Archivo y Fichero tiene una estructura que, en líneas generales, responde a la clasificación y agrupación de la documentación en "mesas", "factores", "carpetas" y "legajos". La configuración de estos niveles de agrupamiento responde a criterios temáticos, geográficos y temporales. Mientras las mesas y factores tienen carácter temático, las carpetas pueden seguir este criterio de ordenamiento u otro geográfico (por localidades) o por años.

Los factores están vinculados a la producción de la información en el territorio, esto es, a la "reunión de información". Los factores "político", "estudiantil”, "religioso", "social”, "subversivo", "gremial”, se constituían en una primera marca o connotación de la información que se aplicaba en la delegación que producía la información. ${ }^{16}$ Con esa categorización la documentación era remitida a la Dirección Central. La estructura del archivo reproduce esa lógica por factores para la clasificación y el ordenamiento de la documentación al interior de algunas mesas. En cierta medida, la estructura del archivo es reveladora de los sujetos sociales, organizaciones y actividades sobre los que la DIPPBA detenía su mirada.

Las mesas y carpetas responden a la diagramación propia del archivo, en tanto niveles de ordenamiento pensados para la clasificación, guarda y acceso de la documentación. Las mismas están identificadas por letras y en algunos casos incluyen factores (mesa A factores estudiantil, político, comunal, Mesa B factor gremial y económico, Mesa De factor religioso y entidades sociales) mientras que en otras, la letra identifica a les sujetes y organizaciones fichadas: Mesa C comunismo, y mesa DS, “delincuente subversivo”.

\section{A QUiÉnes MirABAN, A QUIÉNES Miramos}

Finalmente queremos detenernos en les diferentes sujetes sociales a les que la institución policial miró, persiguió y registró durante la segunda mitad del siglo XX. La creación de la DIPPBA se inscribe en un contexto internacional marcado por la Guerra Fría y por la implementación en América Latina de la llamada Doctrina de la Seguridad Nacional. Al mismo tiempo, en el plano nacional, el escenario político estuvo signado por la proscripción del peronismo. Es decir, en la Argentina el "enemigo interno" no solo estuvo constituido por el comunismo sino por el movimiento peronista proscripto. 
La definición del enemigo interno -que fue cambiando de forma y que debe ser leído en el marco de los distintos contextos históricos y normativa vigente- podía encontrarse en los partidos políticos, en las organizaciones sociales, en los medios de comunicación, en las fábricas, en las escuelas y universidades, en las religiones, en los municipios. Para la DIPPBA, ese enemigo interno podía ser objeto de fichaje o señalado como blanco a ser eliminado.

En este sentido, como señalamos anteriormente, las Mesa C y la Mesa Ds (delincuente subversivo) son reveladoras de la definición del enemigo interno. Mientras que la Mesa $C$ reúne información sobre personas, organizaciones y actividades calificadas por la DIPPBA como comunistas, la Mesa Ds en cambio, tenía por finalidad registrar toda clase de temáticas vinculadas a la actividad calificada/considerada subversiva. El hecho significativo es que en 1970 la Mesa C deja de constituir nuevos legajos, momento en que la Mesa Ds comienza a incrementar exponencialmente su producción documental. La Mesa Ds también es la que en mayor medida da cuenta de la inscripción de la DIPPBA con la comunidad informativa, la que trasluce la relación entre inteligencia y represión, la que amplía su alcance territorial fundiendo sujetos vigilados tanto individuales como colectivos.

El sujeto colectivo o individual vigilade, perseguide y registrade quedó sellado en la estructura de la DIPPBA y en una taxonomía burocrático-represiva que se evidencia en la organización del archivo: gremial, extremista, subversivo, comunista. Sin embargo, en muchos momentos, la realidad permeó aquellas estructuras. Las propias dinámicas burocráticas y la realidad fueron imponiendo coyunturas que son posibles de analizar en la documentación archivada por la DIPPBA. Un minucioso registro sobre las villas miserias de la provincia de Buenos Aires permite ejemplificar. En cada uno de los legajos -cuantiosos en la Mesa Referencia- las delegaciones informaban sobre la existencia o no de referentes y organizaciones sociales. Sin embargo, también realizaban análisis poblacionales y del territorio, excediendo aparentemente un interés político ideológico.

Por otro lado, la propia institución policial y las personas que la componen -en tanto sujetos históricosinevitablemente transparentan sentidos y construcciones sobre la sociedad que trascienden el objeto de la inteligencia. En consecuencia, hay miradas, señalamientos y categorizaciones presentes en la documentación producida que se evidencian a partir de nuevas preguntas y categorías de análisis.

Muchos trabajos de investigación que utilizan como fuente documentos del fondo -incluso algunos elaborados desde la propia CPM- comenzaron a interesarse por la mirada de la inteligencia policial sobre las mujeres, referentes indígenas, poblaciones migrantes, refugiades, la marginalidad social, las diversidades sexuales (Lanteri, Jaschek, Sahade y Sampietro, 2017).

Esos nuevos interrogantes son y serán posibles a partir del trabajo cuidadoso que se realizó sobre los documentos en forma integral desde los principios y herramientas de la disciplina archivística. Reconstruir el contexto de producción de los documentos, conocer sobre su productor, y conservarlos íntegros es lo que nos permite hoy abrir un diálogo con investigadores sociales, de la justicia, de las querellas y de las/los sujetos individuales y colectivos vigilades y perseguides. Y constituir así, una política pública que apueste al acceso y a la difusión para que nuevas miradas, nuevas preguntas y lecturas sean posibles.

\section{REFERENCIAS}

Aguila, A. (2013). Las tramas represivas: continuidades y discontinuidades en un estudio de caso. La Dirección General de Informaciones de la provincia de Santa Fe, 1996-1991. Sociohistórica, (31), 1-26. Recuperado de: https://w ww.sociohistorica.fahce.unlp.edu.ar/issue/view/158

Barreneche, O. (2019). De brava a dura. Policia de la provincia de Buenos Aires. Una historia (1930-1973). Rosario: Prohistoria 
Comisión Provincial por la Memoria (2015). La inteligencia policial a través de sus documentos. Historia institucional de la DIPPBA. Recuperado de https://www.comisionporlamemoria.org/project/historia-institucional-de-la-di ppba/

Da Silva, L. y Jelín, E. (2002). Los archivos de la represión. Documentos, memoria y verdad. Madrid: Siglo XXI Editores.

Funes, P. (2004). Medio siglo de represión. El Archivo de la Dirección de Inteligencia de la Policía de la Provincia de Buenos Aires. Revista Puentes, (11), 34-42. Recuperado de: https://www.comisionporlamemoria.org/archivos /puentes/11puentes.pdf

Funes, P., Jaschek, I. (2005). Dossier documentos. De lo secreto a lo público. Tercera entrega: La creación de la DIPBA. Revista Puentes, (16), 65-74. Recuperado de: http://www.comisionporlamemoria.org.dossier3

Ginzberg, V. (05 de octubre de 2003). El archivo. Página/12. Recuperado de: https://www.pagina12.com.ar/diario/ elpais/1-26339-2003-10-05.html

González Quintana, A. (1998). Los Archivos de la seguridad del Estado de los desaparecidos regímenes represivos. París: UNESCO. Recuperado de: http://www.unesco.org/webworld/ramp/secret_spanish.htlm

Jaschek I., Lanteri M., Sahade J. y Soler E. (2018). Tres estrategias posibles para pensar las políticas de acceso. La CPM y el fondo documental DIPPBA. Hilos Documentales, I (1), e004. Recuperado de: https://revistas.unlp.edu.ar/ HilosDocumentales

Kahan, E. (2007). ¿Qué represión, qué memoria? El archivo de la represión de la DIPBA: problemas y perspectivas. Revista Question, 1 (16). Recuperado de: https://perio.unlp.edu.ar/ojs/index.php/question/article/view/459

Lanteri M., Jaschek I., Sahade J. y Sampietro V. (2017). Diferentes usos de un archivo sensible en 14 años de gestión. La CPM y las consultas al fondo DIPPBA. Ponencia presentada en las XVI Jornadas Interescuelas en Historia (Mar del Plata, Argentina).Recuperado de:https://www.aacademica.org/000-019/609

Marengo, M. E. (2013). Lo aparente como real. Un análisis del sujeto "comunista", en la creación y consolidación del servicio de inteligencia de la policía de la Provincia de Buenos Aires. Aletheia, 3 (6). Recuperado de: http://ww w.memoria.fahce.unlp.edu.ar/art_revistas/pr.6114/pr.6114.pdf

Marengo, M. E. (2018). Los servicios de inteligencia de la Policía de la Provincia de Buenos Aires y la construcción del "sujeto comunista" (1955 - 1962). (Tesis de doctorado). Universidad Nacional de La Plata, La Plata, Argentina. Recuperado de: http://sedici.unlp.edu.ar/handle/10915/75497

Muzzopappa, E. y Mereb, A. (2020). Tramas locales de inteligencia en la zona andina de Río Negro (1975-1980). En M.T. Varela y R. Tarifeño Molina (coord.). La Patagonia en el escenario nacional: presente, pasado y futuro (pp. 374-394). Viedma: UNComa, CURZA. Recuperado de: http://rid.unrn.edu.ar/handle/20.500.12049/5255

Nazar, M. (2018). Secretos, reservados y confidenciales: la producción de las fuerzas armadas y de seguridad como fuente para la historiografía. Estudios Sociales del Estado, 4 (7), 243-264. Recuperado de: http://www.estudioss ocialesdelestado.org/index.php/ese/article/view/151/117

Sahade, J., Lanteri M., Jaschek I. (2016). La inteligencia policial a través de sus documentos. Ponencia presentada en las jornadas Crimen y Sociedad. 10 años de estudio sobre policía, delito y justicia, en perspectiva histórica (San Carlos de Bariloche, Argentina).

\section{Notas}

1 El 4 de enero de 1956 se crea el Servicio de Inteligencia de la Policía de la Provincia de Buenos Aires. A lo largo de su existencia fue cambiando de nombre y rango. La última nominación es de 1991 y es la que se adopta para definir al fondo: Dirección de Inteligencia de la Policía de la Provincia de Buenos Aires (DIPPBA).

2 Ley 12.642/2000, véase: https://normas.gba.gob.ar/documentos/BO8bnckV.html

3 Se aplicó un protocolo de acceso contemplando a los documentos con datos sensibles (según define la Ley de Habeas Data $\left.\mathrm{N}^{\circ} 25.326\right)$.

4 En el 2004, la revista Puentes publicó un artículo de Patricia Funes sobre el archivo. La nota hace hincapié en la amplitud temporal del fondo -en las características de la entidad productora- que no lo asocian exclusivamente a las graves violaciones a los derechos humanos de la década de los 70 (Funes, 2004). 
5 El decreto 2726/83 firmado por Reynaldo Bignone hacia el fin de su gobierno, que ordenaba la destrucción de los documentos de la llamada "guerra sucia", no desalentó a los organismos de DDHH y familiares en la búsqueda de pruebas.

6 Para abordar la historia de la Policía de la Provincia de Buenos Aires en un tramo fundamental del siglo XX véase Barreneche (2019).

7 Sobre los conceptos básicos de la archivística aquí utilizados referirse al articulo de Nazar y García Novarini "Los archivos de inteligencia en Argentina" en este mismo Dossier.

8 La descrición general del fondo documental y el cuadro de clasificación están disponibles en la página web de la CPM: https://www.comisionporlamemoria.org/extra/archivo/cuadroclasificacion/

9 El 29 de diciembre de 1955, por decreto de intervención federal $\mathrm{N}^{\circ} 3603$, se disuelve la Dirección de Orden Público por haber desarrollado "una actividad repudiable" y se designa a une integrante de las Fuerzas Armadas como interventor para reestructurar el organismo. El decreto $\mathrm{N}^{\circ} 3603$, Provincia de Buenos Aires intervención nacional, dice textualmente: "Que es preocupación esencial de esta intervención, el encausamiento de las instituciones provinciales hacia un destino compatible con la tradición democrática, que ha caracterizado a nuestro país desde su nacimiento, reafirmado una vez más por los postulados de la Revolución Libertadora y puestos en ejecución con patriotismo y desinterés por los hombres que la animan; que dentro de esta orientación no es posible admitir la subsistencia de Organismos, Reparticiones o dependencias cuya finalidad inconfesable sea coartar libertades individuales o colectivas, invocando al efecto intereses sociales mediante facultades atribuidas al margen de toda ética administrativa incompatible con la libertad y dignidad humana".

10 Entrevista realizada por el "Noticiero bonaerense" y rescatada por la Filmoteca Online: "Una nueva policía”. https://w ww.youtube.com/watch?v=ufpXXK00vGc\&ab_channel=FilmotecaOnline

11 Entre la documentación previa a la creación de la DIPPBA predominan legajos prontuariales pero también información sobre entidades, federaciones, actos y congresos de personas o entidades calificadas de comunistas. Sobre este tema ver los trabajos de María Eugenia Marengo (2013, 2018).

12 CPM, Fondo DIPPBA, Div. Cen. AyF, Mesa Referencia, legajo 12166.

13 El 11/01/1980 se aprueba el Sistema de Inteligencia de la Policía de la Provincia (S.I.P.P.B.A.) mediante resolución 40.370/80, orden del día 25.199. En el artículo 1 se resuelve: "Crear una nueva estructura informativa (S.I.P.P.B.A.) para la Institución, que satisfará en forma permanente y oportuna las necesidades de información o inteligencia que tengan los diferentes niveles de conducción y ejecución contribuyendo a satisfacer las necesidades del gobierno". En la práctica, lo que intenta el SIPPBA es unificar en un solo sistema informativo, como usuarios o como proveedores, a los componentes de las tres direcciones generales, Seguridad, Inteligencia e Investigaciones; la responsabilidad de la reunión, tratamiento y difusión de la información sigue en la Dirección de Inteligencia. Convierte así a todos los policías en elementos de reunión de información. CPM, fondo DIPPBA, Div Cen Ayf, Mesa Doctrina, legajo 258, asunto: "Orientación para la conducción de la Fuerza, 1979-1980".

14 El denominado "legajo" es la unidad documental mayoritaria del fondo y tiene diversas extensiones, características y lógicas de elaboración que responden a distintos procedimientos administrativos.

15 CPM, fondo DIPPBA, Div Cen Ayf, Mesa Ds, Carpeta Varios, legajo 7454, asunto "Posible infiltración Esc Normal Alte Brown Quilmes". Año 1976.

16 La información clasificada por factores se replica lógicamente en toda la Comunidad Informativa. De ello da cuenta Aguila (2013) en su trabajo sobre la Dirección General de Informaciones de la Provincia de Santa Fe y Muzzopappa y Mereb (2020) sobre la documentación "secreta y confidencial" del municipio de Ingeniero Jacobacci, Provincia de Río Negro, hallada en 1984. 